\title{
NOUVELle
}

\section{Translocation nucléaire dans les neurones en migration : rôle de la sémaphorine 6A et de son récepteur Plexine-A2}

Alain Chédotal, Julie Renaud
Département de Développement, Institut de la Vision, Inserm UMRS 592, 17, rue Moreau, 75012 Paris, France. alain.chedotal@inserm.fr
> Dans la plupart des tissus, des cellules migrent au cours de l'embryogenèse pour atteindre leur destination finale. Ce processus est particulièrement important dans un organe complexe comme le système nerveux dans lequel des anomalies de migration sont à l'origine de nombreuses maladies neurologiques (syndrome de Joubert, lissencéphalie, certaines formes d'épilepsie...) et seraient aussi impliquées dans des maladies comme l'autisme et la schizophrénie [1, 2].

\section{Comment se déplacent les neurones}

Les neurones en cours de migration sont hautement polarisés : ils émettent à l'avant du corps cellulaire un prolongement (qui souvent deviendra l'axone) terminé par un cône de croissance capable de s'orienter dans l'environnement traversé par le neurone, sous l'influence de molécules de guidage [11]. À la base de ce prolongement (que nous appellerons prolongement guide), et à l'avant du noyau, se trouvent l'appareil de Golgi et le centrosome ou centre organisateur de microtubules, un organite clé de la migration cellulaire [2]. Le centrosome est constitué de deux centrioles où sont nucléés les microtubules. Ces derniers s'étendent antérieurement dans le prolongement guide, mais aussi caudalement pour constituer autour du noyau « une cage » qui l'englobe. Pendant la migration, le centrosome (ainsi que le Golgi) s'éloigne du noyau et pénètre dans le prolongement antérieur guide, puis le noyau est tracté vers l'avant par l'intermédiaire de la cage de microtubules, avant qu'un nouveau cycle ne commence. Dans certains neurones, il semble aussi que l'avancée du corps cellulaire soit accompagnée d'une contraction de la cellule à l'arrière du noyau, mettant en jeu la myosine II [3]. Le mouvement du noyau est donc saltatoire et la migration neuronale se résume essentiellement à une translocation du noyau et des organites dans le prolongement guide. Les cellules granulaires du cervelet (ou grains) sont probablement les neurones dont la migration a été la plus étudiée [4]. Les grains naissent à la surface du cervelet dans une région appelée la couche granulaire externe (CGE) puis migrent parallèlement à la surface du cervelet (on parle de migration tangentielle) en émettant deux prolongements qui poussent en direction opposée et deviendront des axones (les fibres parallèles). Dans une seconde phase, les grains émettent un troisième prolongement perpendiculairement à la surface. Ce prolongement va migrer le long des ramifications de cellules gliales spécialisées (la glie de Bergmann) en étant accompagné par le corps cellulaire, pour traverser la couche moléculaire et atteindre son emplacement final, la couche granulaire interne (Figure 1). Cette seconde phase est appelée migration radiaire [5]. Les mécanismes cellulaires et moléculaires contrôlant la migration tangentielle des grains, mais plus particulièrement la transition vers un mode de migration radiaire, étaient méconnus [4].

\section{Le rôle de la Sémaphorine 6A dans la migration des grains}

Nous avions montré il y a quelques années que la Sémaphorine Séma6A, une protéine transmembranaire appartenant à la famille des sémaphorines [6], est spécifiquement exprimée dans la couche granulaire externe, par les grains en migration tangentielle et que son expression s'arrête lorsque débute la migration radiaire [4]. Pour étudier la fonction de cette protéine, nous avions analysé le développement du cervelet de souris déficientes pour la protéine Séma6A, obtenues par recombinaison homologue (une collaboration avec le Dr Kevin Mitchell, Trinity College, Dublin, Irlande). Nous avions ainsi montré que chez les souris n'exprimant pas Séma6A, près de la moitié des grains étaient incapables de quitter la couche moléculaire, restant de manière permanente bloqués à la surface du cervelet. Toutefois, ces grains ectopiques survivaient et se différenciaient normalement. Nous avions dans un second temps analysé la migration des cellules granulaires dépourvues de Séma6A en culture organotypique par imagerie en vidéomicroscopie et montré que l'incapacité des grains à migrer était due à un défaut de translocation du corps cellulaire pendant la migration tangentielle. Toutefois, les causes de cette anomalie à une échelle subcellulaire étaient inconnues.

\section{Plexine A2 contrôle}

le couplage centrosome-noyau au cours de la migration des grains

Nous nous sommes alors intéressés à la plexine A2, l'un des deux récepteurs connus de la Séma6A [7]. Nous avons tout d'abord montré que la plexine A2 est également exprimée par les cellules granulaires en migration tangentielle mais aussi par celles qui migrent de façon radiaire [8]. L'analyse du développement du cervelet chez des souris déficientes pour la plexine A2 (une collaboration avec l'équipe du Dr Hajime Fujisawa, Université de Nagoya, Japon) a révélé des anomalies de 
migration des grains similaires à celles observées chez les souris déficientes en Séma6A. \&n outre, une seconde lignée de souris, mutante pour plexine A2 (obtenue par mutagenèse à l'ENU par le Dr Susan Akermann, Jackson Laboratory, Bar Harbor, États-Unis) et porteuse d'une mutation ponctuelle dans son domaine extracellulaire au niveau du site de liaison de la Séma6A, présente des défauts identiques de migration des grains. Enfin, des expériences de vidéo-microscopie ont mis en évidence qu'en l'absence de plexine A2, la translocation du corps cellulaire était altérée pendant la migration tangentielle. Nous avons mis au point une technique permettant de visualiser les mouvements coordonnés du centrosome et du noyau par imagerie des grains en migration en leur faisant exprimer une protéine enrichie au niveau du centrosome, la centrine, fusionnée à la protéine fluorescente verte (GFP). Cette approche a permis de montrer que le blocage de la translocation nucléaire dans les grains déficients en plexine A2 était dû à un découplage du noyau et du centrosome, ce dernier pouvant parfois s'éloigner à une distance supérieure à $30 \mu \mathrm{m}$ contre 5-7 $\mu \mathrm{m}$ normalement. De plus la polymérisation de l'actine est également anormale en l'absence de plexine-A2, entraînant l'apparition de nombreux filopo- des à la surface des grains. De manière surprenante, l'absence de translocation du noyau dans les grains déficients en Séma6A semble être pour sa part corrélée à une absence de découplage entre noyau et centrosome [8]. Ces données mettent donc en évidence un nouveau rôle des molécules de guidage axonal, les sémaphorines, dans la migration neuronale, via une modulation du couplage noyaucentrosome. II reste toutefois à expliquer ces anomalies de migration à un niveau moléculaire.

\section{Centrosome et maladies neurologiques} Ces résultats sont également à rapprocher d'autres travaux qui ont révélé l'importance des protéines du centrosome dans la migration neuronale ainsi que leur lien avec des maladies neurologiques $[1,2]$ et des plexines de type $A$ dans la migration neuronale radiaire dans le néocortex [9]. Il est intéressant de souligner que plusieurs études récentes de génétique humaine associent le gène plexine A2 (ou PLXNA2) à l'autisme et la schizophrénie [10]. $\diamond$

\section{Nucleus translocation}

in migrating neurons:

key control by Sema6A and plexin A2

\section{REMERCIEMENTS}

Ce projet a été soutenu par une subvention «ANR Neurosciences ».

\section{RÉFÉRENCES}

1. Tsai LH, Gleeson JG. Nucleokinesis in neuronal migration. Neuron $2005 ; 46: 383-8$.

2. Higginbotham HR, Gleeson JG. The centrosome in neuronal development. Trends Neurosci 2007 ; 30 : 276-83.

3. Bellion A, Baudoin JP, Alvarez C, et al. Nucleokinesis in tangentially migrating neurons comprises two alternating phases : forward migration of the Golgi/centrosome associated with centrosome splitting and myosin contraction at the rear. J Neurosci 2005 ; 25 : 5691-9.

4. Kerjan G, Dolan J, Haumaitre C, et al. The transmembrane semaphorin Sema6A controls cerebellar granule cell migration. Nat Neurosci 2005 ; 8 : 1516-24.

5. Solecki DJ, Govek $\varepsilon \varepsilon$, Hatten ME. mPar6 alpha controls neuronal migration. J Neurosci 2006 ; 26, 10624-5.

6. Nasarre P, Constantin B, Drabkin HA, Roche J. Sémaphorines et cancers : état des lieux. Med Sci (Paris) 2005; $21: 641-7$

7. Suto F, Tsuboi M, Kamiya $H$, et al. Interactions between Plexin-A2, Plexin-A4, and Semaphorin 6 A control laminarestricted projection of hippocampal mossy fibers. Neuron 2007 ; 53 : 535-47.

8. Renaud J, Kerjan G, Sumita I, et al. Plexin-A2 and its ligand, Sema6A, control nucleus-centrosome coupling in migrating granule cells. Nat Neurosci $2008 ; 11: 440-9$.

9. Chen G, Sima J, Jin M, et al. Semaphorin-3A guides radial migration of cortical neurons during development. Nat Neurosci $2008 ; 11: 36-44$

10. Wray NR, James MR, Mah SP, et al. Anxiety and comorbid measures associated with PLXNA2. Arch Gen Psychiatry 2007 ; 64 : 318-26.

11. Mehlen P, Rama N. Nétrine-1 et guidage axonal: signalisation et traduction asymétrique. Med Sci (Paris) $2007 ; 23: 311-5$.

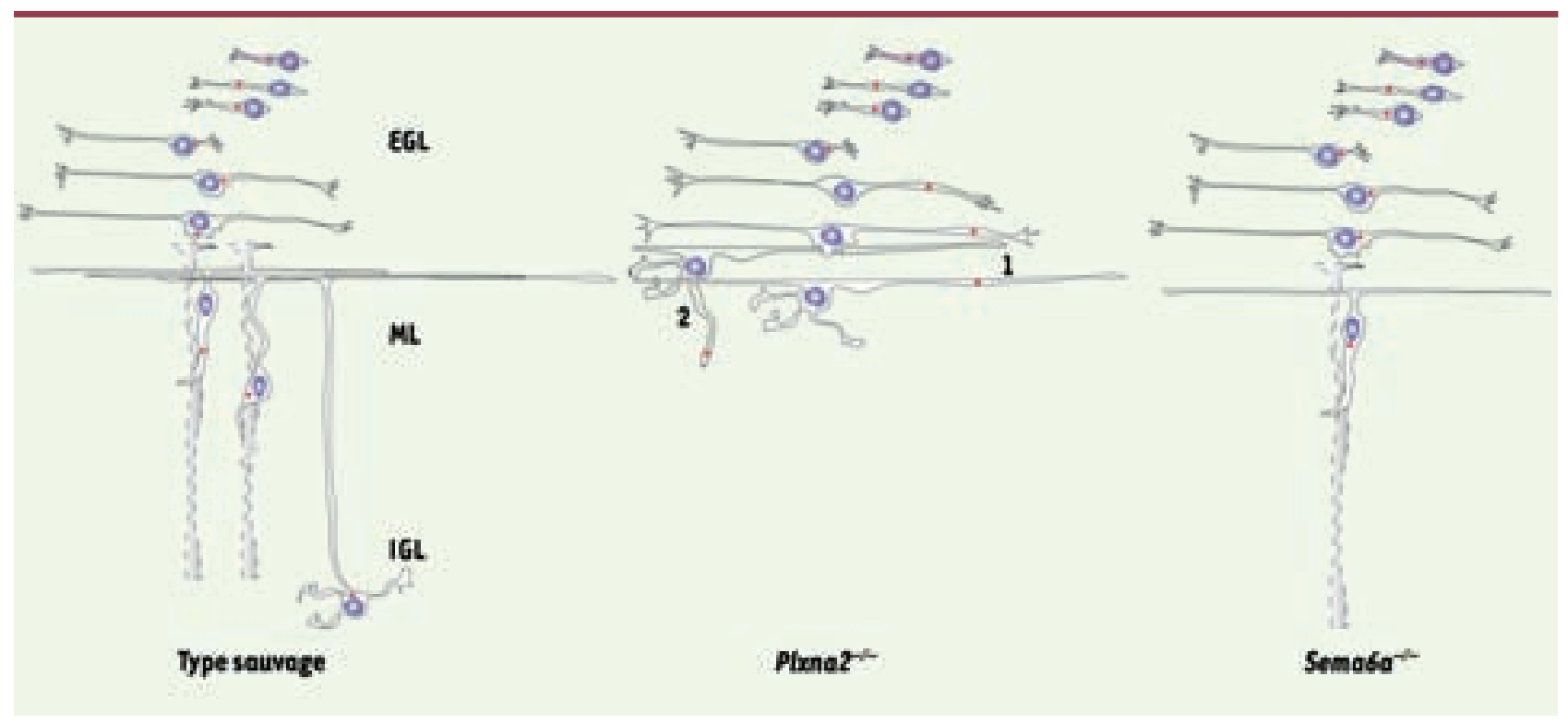

Figure 1. Cellules granulaires du cervelet. Les grains naissent à la surface du cervelet dans une région appelée la couche granulaire externe puis migrent parallèlement à la surface du cervelet en émettant deux prolongements qui poussent en direction opposée et deviendront des axones. Dans une seconde phase, les grains émettent un troisième prolongement perpendiculairement à la surface. 